\title{
Signal-to-noise ratios of teleseismic receiver functions and effectiveness of stacking for their enhancement
}

\author{
Igor B. Morozov ${ }^{1}$ and Kenneth G. Dueker \\ Department of Geology and Geophysics, University of Wyoming, Laramie, Wyoming, USA \\ Received 4 December 2001; revised 2 August 2002; accepted 30 September 2002; published 19 February 2003.
}

[1] We present a method for the measurement of spatially variable signal-to-noise $(\mathrm{S} / \mathrm{N})$ ratios in multichannel teleseismic receiver function (RF) images. The $\mathrm{S} / \mathrm{N}$ ratio is defined as a measure of coherency of the final image, and the approach is applicable to any RF imaging technique that employs mapping of the records into depth followed by their summation as the final signal enhancement step. In such methods, all of the converted phases become horizontally aligned in the depth domain, and their coherent (signal) and incoherent (noise) components can be estimated by using stacking statistics. For 10 locations along two subarrays of the Continental Dynamics of Rocky Mountains Project (CD-ROM) teleseismic array, after a limited RF editing, we apply our method to the image resulting from three-dimensional (3-D) prestack RF depth migration. The resulting amplitude $\mathrm{S} / \mathrm{N}$ values to vary from 0.1 to 0.3 in the individual RFs and from 1 to 5 in the final image, with significant spatial variability. These moderate $\mathrm{S} / \mathrm{N}$ values argue in favor of sampling redundancy achieved in array recording and multichannel RF processing. In order to further reduce noise in RF images, denser and longer deployments or additional signal enhancement techniques are advisable. Because of its ability to provide direct assessment of image quality in three dimensions, including its dependence on the frequency band and data coverage, our method can also be potentially used for real-time array tuning and image focusing required for moving arrays proposed for the USArray program. INDEX TERMS: 7218 Seismology: Lithosphere and upper mantle; 7260 Seismology: Theory and modeling; 7294 Seismology: Instruments and techniques; KEYWORDS: receiver functions, depth imaging, migration, image quality

Citation: Morozov, I. B., and K. G. Dueker, Signal-to-noise ratios of teleseismic receiver functions and effectiveness of stacking for their enhancement, J. Geophys. Res., 108(B2), 2106, doi:10.1029/2001JB001692, 2003.

\section{Introduction}

[2] Teleseismic receiver functions (RF) are broadly used for imaging the Moho and mantle discontinuities [e.g., Vinnik, 1977; Ammon et al., 1990; Shearer, 1991; Bostock, 1996; Dueker and Sheehan, 1998; Shen et al., 1998; Chevrot et al., 1999; Gurrola and Minster, 2000; Lewis et al., 2001]. With the advent of portable Program for Array Seismic Studies of the Continental Lithosphere (PASSCAL) broadband arrays [e.g., Dueker and Sheehan, 1998; Neal and Pavlis, 1999; Bostock and Rondenay, 1999; Rondanay et al., 2001] the volumes of RF data sets have increased dramatically, and detailed RF imaging of the crust and upper mantle has improved. Several source and receiver array imaging techniques were developed, such as common conversion point stacking [Dueker and Sheehan, 1998; Zhu, 2000], velocity spectrum stacking [Gurrola et al., 1994; Shen et al., 1998], $\tau-p$ record interpolation [Neal and

\footnotetext{
${ }^{1}$ Now at Department of Geological Sciences, University of Saskatchewan, Saskatoon, Saskatchewan, Canada.

Copyright 2003 by the American Geophysical Union. 0148-0227/03/2001JB001692\$09.00
}

Pavlis, 1999], and prestack depth migration [e.g., Bostock and Rondenay, 1999; Sheehan et al., 2000; Bostock et al., 2001; Poppeliers and Pavlis, 2003a, 2003b]. All of these techniques ultimately rely on record summation (stacking) for noise reduction. However, the problem of quantitative characterization of the performance of such noise reduction still has not received sufficient attention.

[3] Measurements of noise in RF stacks are often performed by pixel-by-pixel estimation of mean values and variances of the image [e.g., Zhu, 2000]. Such simple measures, however, do not take into account the fact that seismic signal is not simply a collection of independent sample values but a waveform, i.e., a band-limited, mutually correlated time series representing a continuous physical process. From a single time sample recorded at a number of channels, it is impossible to distinguish a signal from some realization of random noise. The only way to identify the signal is to look for some common waveform that is coherent across the channels. Such an approach to multichannel signal-to-noise ratio $(\mathrm{S} / \mathrm{N})$ estimation is conventional in reflection seismics [e.g., Rietsch, 1980], and this is the point of view that we are taking here.

[4] Significant levels of noise in RF records has been recognized, particularly at higher frequencies [e.g., Abers, 


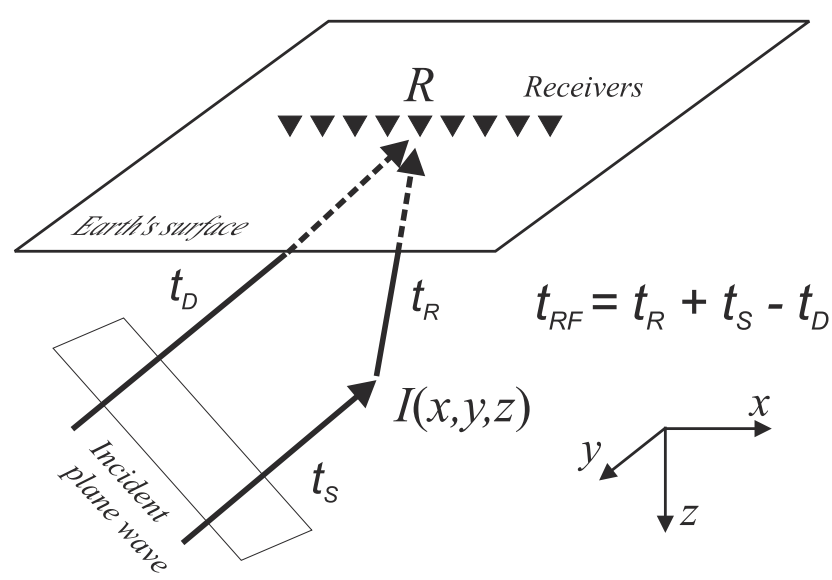

Figure 1. Time-to-depth mapping of a $P d S$ mode used in our analysis. This mapping scheme is also used in Kirchhoff prestack depth migration. For each of the incident $P$ waves, its travel times $\left(t_{S}\right)$ to every point within the model are precomputed and stored. For each of the receivers, $\mathbf{R}$, the $S$ wave travel times to every point of the model are also precomputed. Further, for each RF record and imaging point $I(x, y, z)$, the corresponding travel time maps are combined to form the predicted RF time, $t_{R F}(x, y, z)\left(t_{D}=t_{S}(\mathbf{R})\right.$ is the time of the primary arrival): For any surface location $(x, y)$, the inverse of this function yields the desired mapping: $t_{R F} \rightarrow z$. The travel times are modeled in a (in general) 3 -D $\left(V_{P}, V_{S}\right)$ velocity distribution using an eikonal travel time solver.

1998; Langston and Hammer, 2001], and record summation is broadly used to suppress noise. However, there still remain several outstanding problems in RF imaging, and these problems make the RF case significantly more complex than its counterpart in reflection seismics: (1) the accuracy of the layered Earth model that underlies RF imaging; (2) the accuracy of deconvolution; (3) RF editing, including a selection of the frequency band (also degrading the accuracy of deconvolution); (4) the ability to resolve and suppress other modes interfering with the $P d S$ conversions or to perform multimode imaging; and (5) effects of scattering not accounted for by the conventional RF kinematics (e.g., $P \rightarrow R g, P g, L g, P n, S n$ ) from crustal and potentially mantle heterogeneities. With $P d S$ amplitudes at the level of $2-4 \%$ of the primary $P$ wave, these problems pose significant obstacles to judicious interpretation. Also, RF depth images always represent results of intricate processing (editing, deconvolution, filtering, amplitude correction, binning, stacking, smoothing, etc., often dubbed as "routine" in geological publications), and the effects of this processing on image fidelity may be difficult to assess.

[5] In this study, we do not deem possible answering the questions above; neither can we propose an ideal imaging approach. Instead, by utilizing the multirecord character of most of the existing imaging schemes, we propose a simple and general statistical test of the consistency of the images they create. Therefore, for a given data set and a multichannel RF imaging procedure (and, consequently, for some answer to the problems 1-5 above), a number of questions critical for interpretation can be answered: What are the noise levels in the records contributing to an RF image?
How many records need to be stacked for a meaningful interpretation? How efficient is stacking and is it the optimal procedure for the attenuation of noise? Finally, and most importantly, what is the level of noise remaining in the resulting images? In this context, the "noise" is simply anything that is not consistent with the image presented.

[6] Our definition of the $\mathrm{S} / \mathrm{N}$ ratio focuses specifically on assessment of image quality resulting from a summationbased imaging technique and differs from the measures of recording channel noise often employed [e.g., Poppeliers and Pavlis, 2003b]. The exact type of imaging method is not essential; the only requirement is its separation into two steps: (1) individual transformations of each of the input records (typically, sampled in time) into the imaged quantity at the point of interest (e.g., $P$ to $S$ wave scattering amplitude at depth), and (2) summation of the resulting records pertaining to the same imaging (e.g., depth) point in order to enhance the desired signal. Most of the existing multichannel imaging methods fall into this general category of time-to-depth mapping (Figure 1) and subsequent record summation in the depth domain. Among the wellknown examples of such methods are the prestack depth migration and common conversion point stacking.

[7] With the imaging procedure subdivided into two steps, all the variability of approaches (e.g., numerous variants of prestack depth migration) is contained in timeto-depth mapping (Figure 1). This step can also be viewed as an application of a "deterministic" background model reflecting what is known about the kinematics and dynamics of the phase of interest. By contrast, the final summation in the depth domain represents an attempt to attenuate any kind of noise that is not consistent with this model, and its performance can and should be studied independently. By definition, the input to such summation is expected to consist of records containing an identical signal, since all of them relate to the same imaging point (e.g., to the common $P$-to- $S$ wave conversion point). Some imaging approaches, such as the one by I. B. Morozov and K. G. Dueker (Depth-domain processing of teleseismic receiver functions and generalized three-dimensional imaging, submitted to Bulletin of the Seismological Society of America, 2002, hereinafter referred to as Morozov and Dueker, submitted manuscript, 2002), take advantage of such an explicit two-step imaging and allow introduction of coherency filters, signal detectors, visualization and other tools in place of this final stacking procedure. Our $\mathrm{S} / \mathrm{N}$ measurement procedure is also designed as such a "plug-in" used in place of the final depth record summation in three-dimensional (3-D) prestack depth migration.

[8] While emphasizing its applicability to virtually any $\mathrm{RF}$ depth imaging scheme (given its appropriate, modular, implementation (cf. Morozov and Dueker, submitted manuscript, 2002), for the following examples and discussion we need to focus on a specific method. As such, we choose the Kirchhoff depth migration of RFs, in which the time-todepth mapping is designed to convert the $P d S$ conversion times into depth (Figure 1). We use the "diffraction stack" migration, in which the amplitudes of the original time domain RFs are retained upon their conversion to depth. After such mapping, the $P d S$ converted phases become horizontally aligned in depth domain regardless of the underlying mantle structure (yet assuming a correct back- 

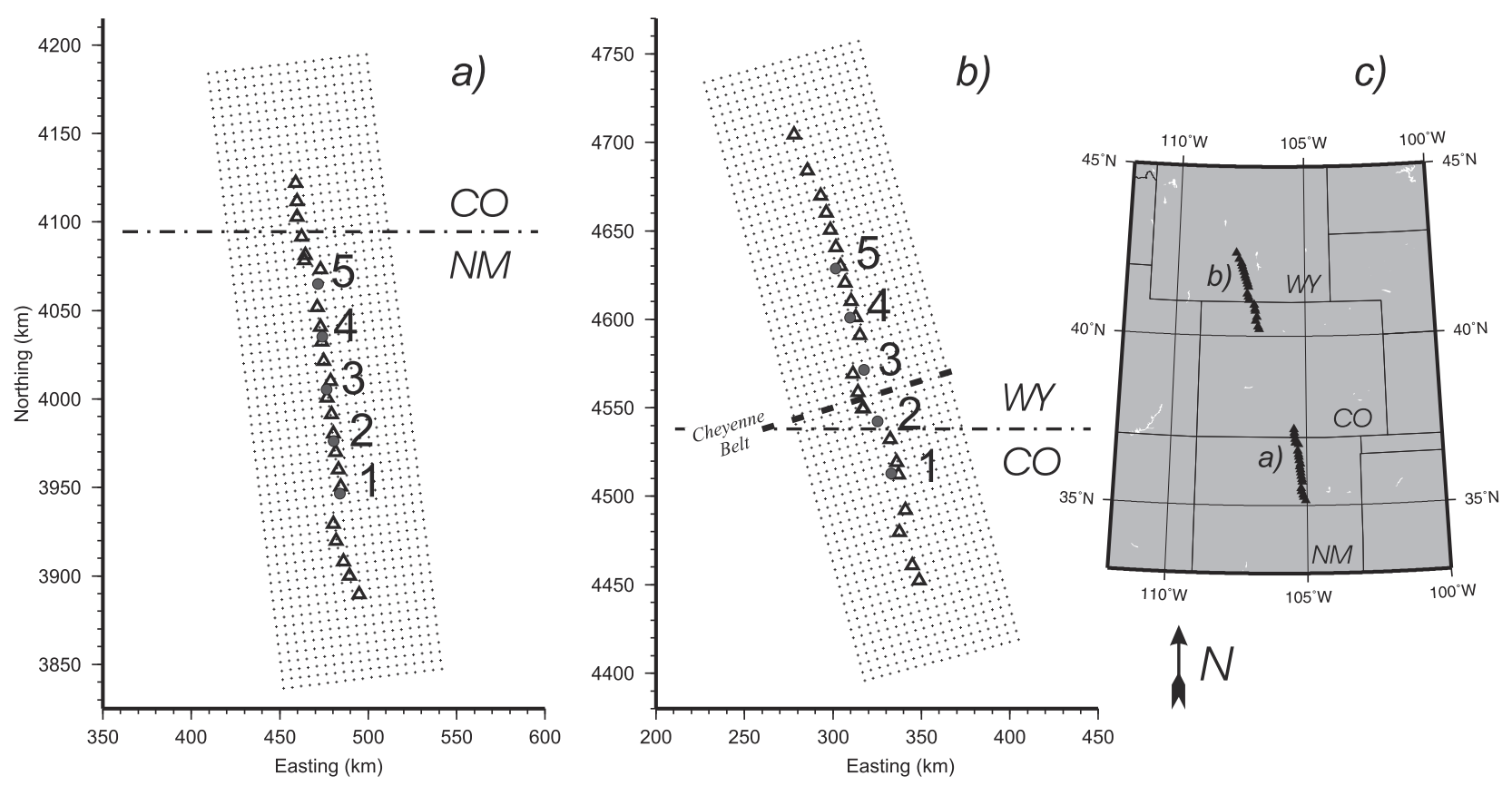

Figure 2. (a) Southern and (b) northern parts and (c) location map of the CD-ROM array. The approximate location of the Cheyenne Belt is shown with a thick dashed line in plot Figure $2 \mathrm{~b}$. The rectangular 3-D imaging grids covering the subarrays are also shown. Five nodes along the axis of each grid are used in our $\mathrm{S} / \mathrm{N}$ estimation (highlighted in gray and labeled 1-5). Coordinates in Figures $2 \mathrm{a}$ and $2 \mathrm{~b}$ are $\mathrm{UTM}$, in kilometers.

ground velocity model [cf. Al-Yahya, 1989]; note, however, that because of low sensitivity of RF moveouts to velocity perturbations, the effect of not accurately known background velocity is insignificant in RF imaging above $\sim 200 \mathrm{~km}$ ). Therefore, in our definition, the $\mathrm{S} / \mathrm{N}$ ratio measures the proportion of coherent signal that stacks to form the image, as opposed to multiples and other phases inconsistent with the $P d S$ kinematics and also to the noise caused by scattering and microseisms.

[9] Using the data from the teleseismic Continental Dynamics of Rocky Mountains Project (CD-ROM) experiment [Dueker et al., 2001] that represents a "typical" present-day linear broadband PASSCAL deployment, we demonstrate that noise is still significant in the resulting RF images. It appears that in order to improve the reliability of imaging, we need to employ signal processing techniques that are more effective than stacking in noise suppression. Furthermore, since the RF image $\mathrm{S} / \mathrm{N}$ ratios vary laterally and with depth and are relatively easy to measure, monitoring these ratios (perhaps even during data acquisition) could potentially be used for real-time array adjustment and targeting during extended periods of deployment.

\section{Signal-to-Noise Ratios in Receiver Function Data}

[10] Since the RF signal is influenced by a number of processes that are difficult to control (crustal scattering, site effects, contamination from multiples, deconvolution, filtering) statistical properties of the useful $P d S$ signal and noise are not well understood. Therefore the following estimates are based on a simplified model of a constant amplitude signal embedded in a background of completely uncorre- lated (although band-limited), constant-power noise. This paradigm underlies most of stacking-based signal enhancement schemes (for its more sophisticated variant allowing variable signal and noise amplitudes, see Rietsch [1980]; Morelli and Dziewonski [1987] applied a related S/N estimation method in earthquake seismology). In the case of RF imaging, this paradigm is highly approximate, and therefore the following $\mathrm{S} / \mathrm{N}$ calculations should be interpreted as order-of-magnitude estimates.

[11] In unweighted stacking, multiple recordings are assumed to have the same amplitude of "signal", $s(t)$, and the same noise power, $n^{2}(t)$, in all the channels. In contrast, the noise $n_{i}(t)$ is assumed to be uncorrelated in the different records $i$ and $j(i \neq j):\left\langle n_{i}(t) n_{j}(t)\right\rangle=0$ and with the signal: $\left\langle n_{i}(t) s(t)\right\rangle=0$. Here, angle brackets indicate the average over an ensemble of realizations of the random processes $s(t)$, $n_{i}(t)$. In practice (yet another simplifying assumption), this averaging is replaced with time averaging [e.g., Rietsch, 1980]. For a stack

$$
S(t)=\sum_{i=i}^{N}\left(s(t)+n_{i}(t)\right)=N s(t)+\sum_{i=i}^{N} n_{i}(t),
$$

its mean power can be expressed as

$$
\left\langle S^{2}(t)\right\rangle=N^{2}\left\langle s^{2}(t)\right\rangle+\sum_{i=i}^{N}\left\langle n_{i}^{2}(t)\right\rangle=N^{2}\left\langle s^{2}(t)\right\rangle+N\left\langle n_{i}^{2}(t)\right\rangle .
$$

With increasing number of records $N$, the signal power in the stack grows as $N^{2}$ while the noise power increases linearly resulting in a $\sqrt{N}$ increase in the amplitude signal- 


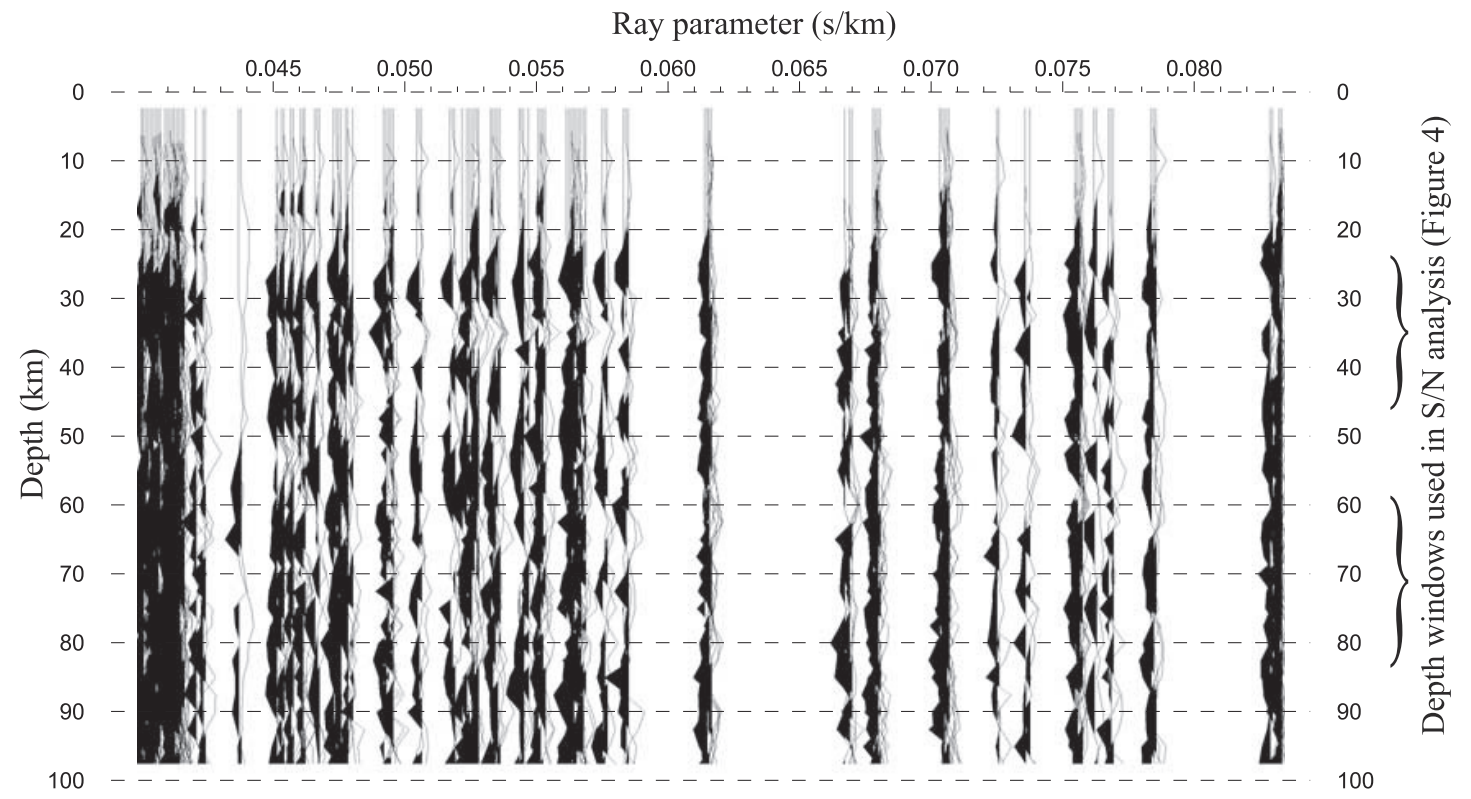

Figure 3. Common image gather (CIG) for the center of the southern imaging grid (large gray dot labeled 3 in Figure 2a). A stack of these records would constitute the traditional prestack depth migrated image at this point. To illustrate their actual variability, the records are not binned, with multiple records plotted at the same values of ray parameter.

to-noise ratio. Note that the noise power in the stack does not decrease as it is sometimes assumed. Rewriting relation (2) in terms of the mean stack power,

$$
\frac{\left\langle S^{2}(t)\right\rangle}{N}=N\left\langle s^{2}(t)\right\rangle+\left\langle n_{i}^{2}(t)\right\rangle
$$

we obtain a simple estimate of the signal-to-nose ratio, both in the original data and in the stack. In the presence of coherent signal, the mean stack power exhibits a linear dependence on $N$. With a sufficiently large number of records available for stacking, this behavior can be tested by random resampling (jack-knifing) the data set and fitting a line to $\left\langle S^{2}\right\rangle / N$ as a function of $N$. The intercept of this line would equal $\left\langle n^{2}\right\rangle$ and the slope equal $\left\langle s^{2}\right\rangle$ (equation (3)).

[12] Before the mean stack power (3) can be computed from RF data, travel time moveouts due to the differences in ray parameters, backazimuths, and relative station positions must be removed from the data, so that the coherent, $s(t)$, component of the records can be regarded as identical in all the channels. A general way to perform such transformation is to convert the records from time to depth at a specified imaging point (Figure 1). This is commonly done in prestack depth migration (depending on the migration scheme employed, this step can also include amplitude corrections [cf. Bostock and Rondenay, 1999]). As a result of such mapping, the records are transformed into depth, $s(t) \rightarrow s(z)$ and form an analog of the common image gather (CIG) that is commonly used for velocity analysis in reflection seismology [e.g., Al-Yahya, 1989]. The CIG essentially shows the contribution of each of the individual $\mathrm{RF}$ records to a depth column under a single surface location, and therefore, ideally, all the true $P d S$ mode conversions become identical, thereby satisfying our model in equation (2). Any other energy inconsistent with the $P d S$ conversion kinematics is misaligned in the $\mathrm{CIG}$ and is treated as noise.

[13] We apply the above technique to RF data from a broadband array of the Continental Dynamics of the Rocky Mountains (CD-ROM) experiment acquired from June 1999 to June 2000. Each of the two CD-ROM deployments consisted of 21 stations, spaced at about 10-12 km (Figure 2). These arrays are among the several large and dense PASSCAL deployments operated recently and provide good data sets for detailed analysis of the upper mantle; and they could be considered "typical" linear arrays for our $\mathrm{S} / \mathrm{N}$ study. For our measurements, we migrate all the records into each of the five selected points in each subarrays (gray dots in Figure 2), using the IASP91 velocity model. Although this velocity model is $1-\mathrm{D}$, we used 3-D ray kinematics taking into account the actual locations of the stations, ray parameters, and backazimuths.

[14] Important to this study is an assessment of what receiver functions should be included in our analysis. To do this, we proceed through a sequence of data culling procedures. First, all direct $P$ wave arrivals between $28^{\circ}$ and $105^{\circ}$ with magnitudes greater than 5.5 are windowed. From this initial data set, receiver functions are constructed for a range of deconvolutional water level parameters. Three different water levels of $0.001,0.01$, and 0.1 are used. Next, the RMS of each trace is calculated and a histogram of the RMS values is made for the three different water level data sets. These histograms are all well peaked around 0.2 with a long tail that extends upward. The receiver function data sets are then sorted by RMS and plotted. Visual inspection of the three data sets is performed to find an appropriate RMS cutoff. Our criterion for what constitutes a usable versus unusable receiver function is based upon the following: (1) the zero-lag arrival (i.e., the $P$ wave arrival) must be the largest arrival on each trace; (2) the trace must not have 


\section{Southern CD-ROM line}
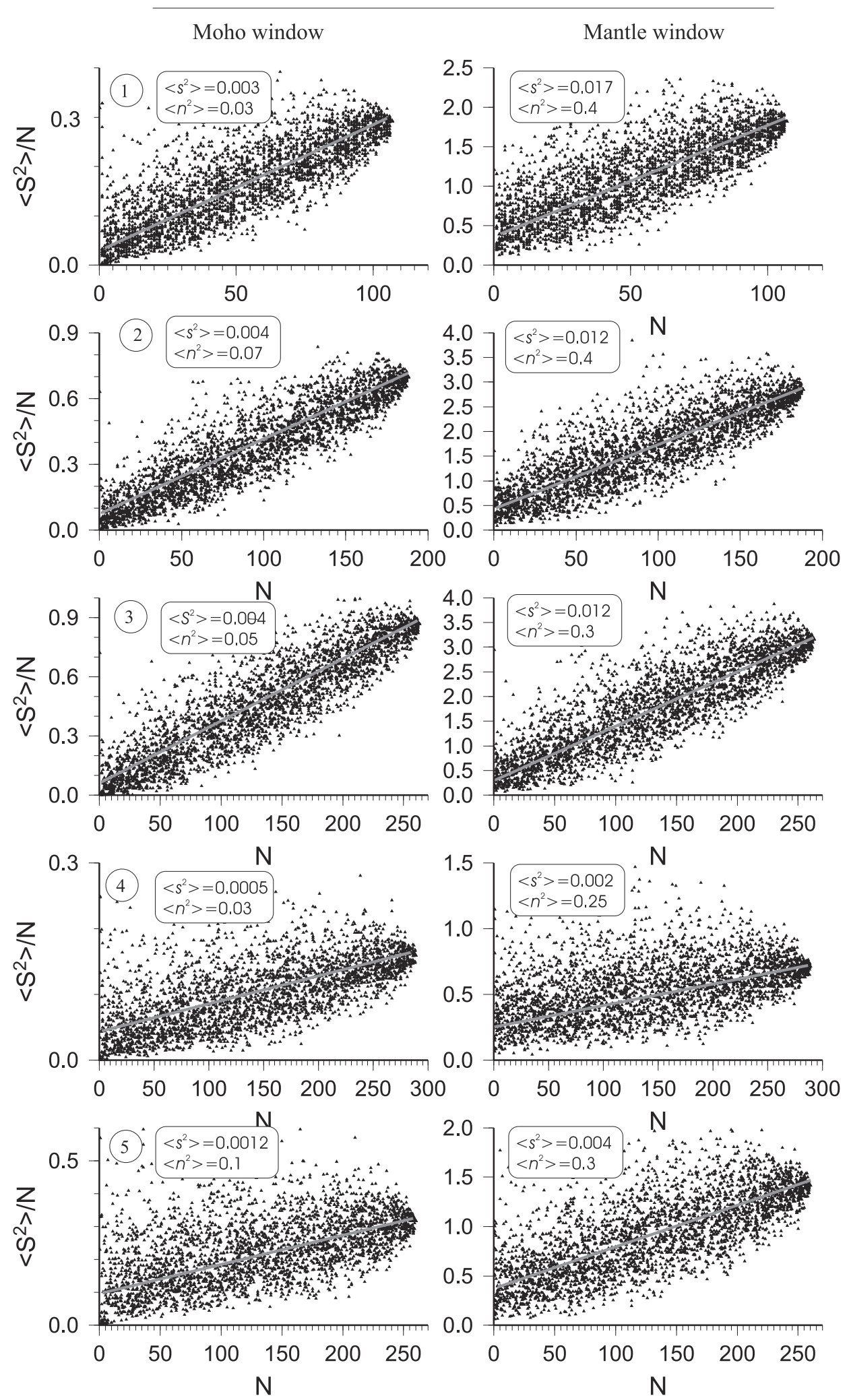

Figure 4. Dependence of the mean stack power (3) on the number of stacked depth-converted records within two depth windows (Figure 3) for the Moho $P d S$ conversion and for a window between 70 and $100 \mathrm{~km}$ depth, measured for five imaging points (A-E) along the axis of each of the grid (Figure 2). The slopes of the axes of these distributions (gray dashed lines) provide estimates of mean signal power, $\left\langle s^{2}\right\rangle$, and their widths correspond to noise power, $\left\langle n^{2}\right\rangle$ (labeled). The units of RF amplitudes are relative. 


\section{Northern CD-ROM line}

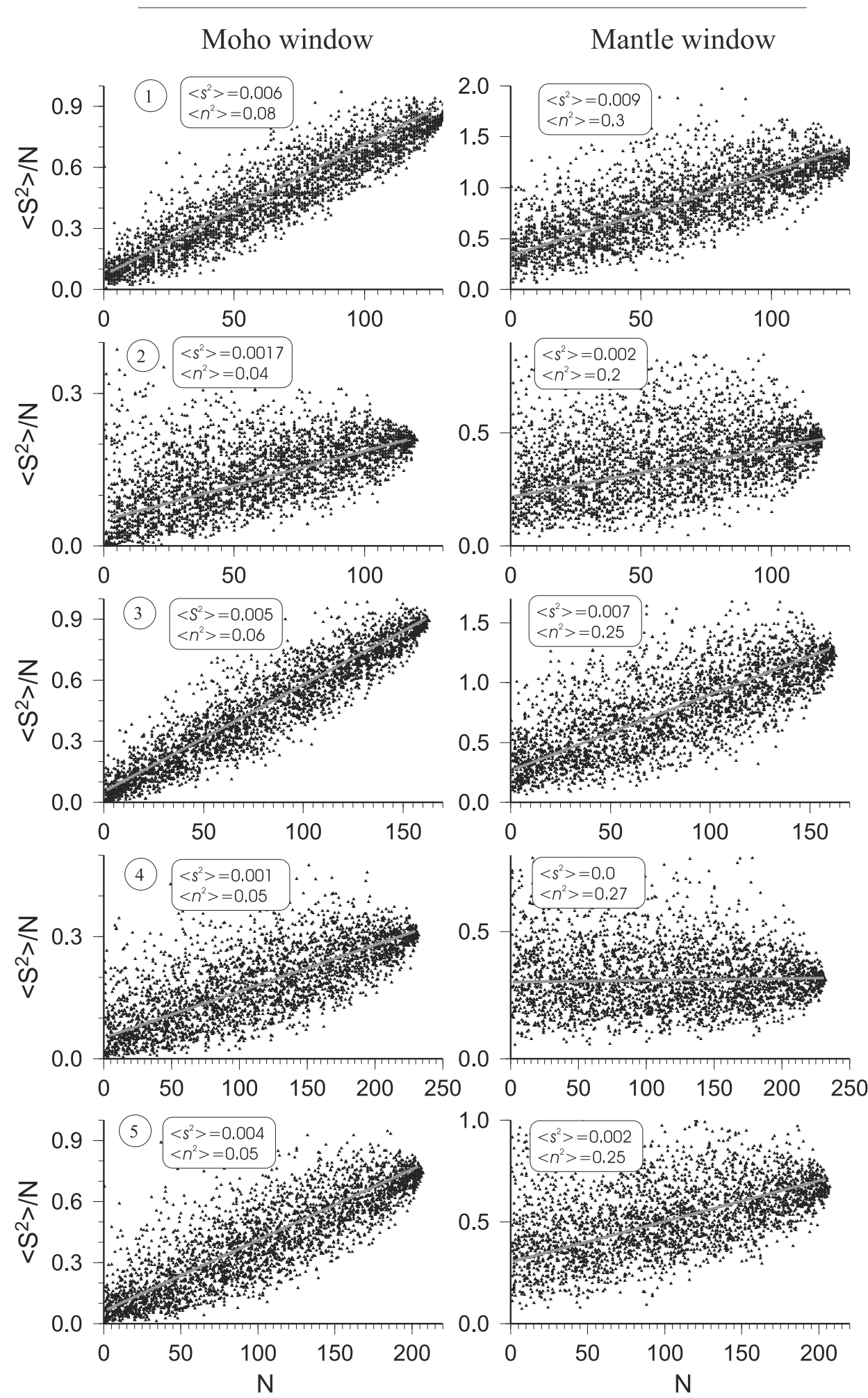

Figure 4. (continued)

monochromatic spectra; and (3) no arrivals larger than the Moho arrival, between 4 and $6 \mathrm{~s}$, are present. After review of the three data sets, we found that the qualitatively "best" data set that satisfies these criteria is associated with the
0.01 water level data set with an RMS cutoff of 0.3 [Dueker and Sheehan, 1998]. This procedure resulted in the removal of $30 \%$ of original data leaving us with 1800 receiver functions for this study. Given the 44 stations in this 
Average $\mathrm{S} / \mathrm{N}$ in individual RFs

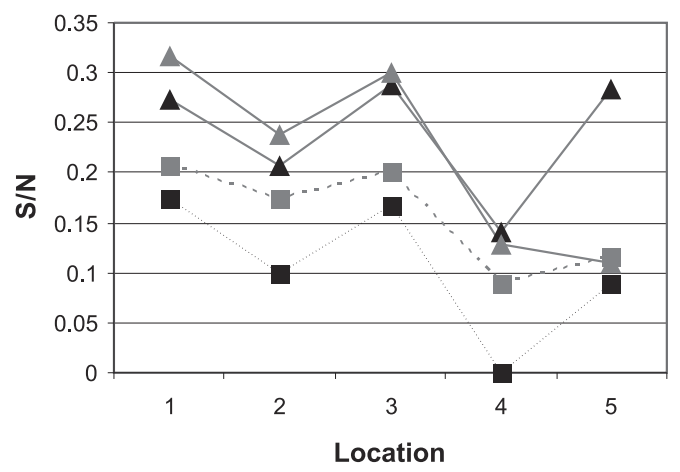

$\mathrm{S} / \mathrm{N}$ in migrated image

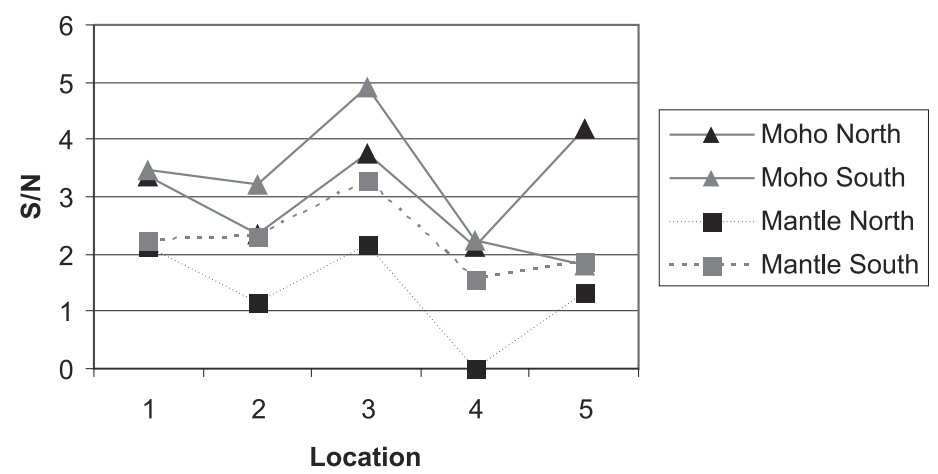

Figure 5. Amplitude $\mathrm{S} / \mathrm{N}$ ratios determined from the signal and noise estimates in Figure 4. Note the variability of the values. Apart from one outlier, the values are within the range $0.1-0.3$, with higher values for the Moho.

experiment that operated for a total of 320 days, our acquisition rate of useful receiver functions is one for every 8 days. This is consistent with our average acquisition rate for similar PASSCAL broadband arrays.

[15] The edited RFs were further filtered using a zerophase band-pass filter between 0.3 and $2.0 \mathrm{~Hz}$ with one octave filter roll-off at both ends. This frequency band is the high $\mathrm{S} / \mathrm{N}$ passband for the CD-ROM data set [Dueker et al., 2001]. The resulting filtered RFs were visually inspected for a minimal waveform distortion, as required for correct timing and amplitude estimates [Chevrot and Girardin, 2000]. For our S/N prestack depth migration measurements, we selected from this set only the records from the stations within $30 \mathrm{~km}$ distance from the imaging points because these stations were considered the best contributors to the image above the depth of $\sim 100 \mathrm{~km}$. From this set of migrated RFs, we extracted 3000 random subsets of $N$ records each, where $N$ was uniformly distributed between 0 and 120-270 (depending on the available coverage of the imaging point). Finally, we stacked the subsets, measured the resulting stack power within two depth windows (Figure 3), and created scatter diagrams (Figure 4).

[16] The distributions of $\left\langle S^{2}\right\rangle / N$ in Figure 4 show linear trends indicating the presence of both coherent signal and noise in the data. By fitting straight lines to these distributions, we estimate the signal power $\left\langle s^{2}\right\rangle$ from the slopes of these lines, and noise power $\left\langle n^{2}\right\rangle$ from their intersections with the axis $N=0$.

[17] The resulting values suggest amplitude $\mathrm{S} / \mathrm{N}$ (coherency) values that are about $0.15-0.3$ for the Moho and $0-$ 0.2 for the mantle between 60 and $80 \mathrm{~km}$ depth, respectively, and exhibit significant spatial variability (Figure 5). The levels of coherency in the final migrated image vary by a factor of $\sim 2$ within $30-60 \mathrm{~km}$ distances. The mantle S/N values are consistently lower than those of the Moho, and both are correlated with the locations. It is not clear why the spatial variations in the $\mathrm{S} / \mathrm{N}$ values appear to be similar for both subarrays. (Figure 5). Such moderate $\mathrm{S} / \mathrm{N}$ values and their spatial variability emphasize the importance of using multichannel recording multiple recordings to improve the image, and also of monitoring $\mathrm{S} / \mathrm{N}$ attributes for selection of imaging techniques, interpretation, and potentially for array design in the future.

\section{Discussion and Conclusions}

[18] With $\mathrm{S} / \mathrm{N}$ ratios ranging between 0.1 and 0.3 (Figure 5), stacking of 10 to 100 records is required in order to bring the coherent signal to the level of noise, and twice as many are probably needed to ensure statistical repeatability of the resulting image. Such RF density is close to the limit of data redundancy of the CD-ROM data set, where we estimate the $\mathrm{S} / \mathrm{N}$ in the final stack ranging between 1 and 5 (1-3 for the subcrustal mantle), with some outliers dropping below this range (Figure 5). Therefore, assuming that signal amplitude consistently exceeding the incoherent noise by a factor of about 3 is desirable for robust imaging, we suggest that several times longer-duration and/or denser deployments are needed compared to that of the CD-ROM. Note, however, that a mere increase in RF data volumes still would not guarantee the prevalence of $P d S$ mode conversions in the resulting image since crustal scattering and filtering/deconvolution artifacts might also produce repeatable waveforms. Crustal scattering may be a formidable problem in RF imaging requiring further careful studies and specially designed experiments.

[19] Our technique of spatially variant depth domain $\mathrm{S} / \mathrm{N}$ estimation provides a direct test of the performance of depth record summation as the signal enhancement method in CCP stacking or prestack depth migration. As our results above suggest, with the existing data sets, both techniques may lead to images that are only moderately above the noise level. One way to overcome these shortcomings is in using much denser, 3-D arrays and longer recording times; however, this approach meets with obvious limitations of the available resources. An alternative approach would be to replace stacking (that is an almost invariable signal enhancement step in almost any imaging algorithm) with more robust signal detection methods. Morozov and Dueker (submitted manuscript, 2002) present a prototype of 3-D prestack depth migration allowing any other type of processing in depth domain, such as median or coherency filtering. Adaptive "dip filtering" in depth domain (Moro- 
zov and Dueker, submitted manuscript, 2002) could also attenuate the coherent, source-induced noise and filtering artifacts that may be difficult to distinguish from $P d S$ conversions because of the limited recording aperture. Another broadly used approach to $\mathrm{S} / \mathrm{N}$ enhancement relies on careful selection of RFs for imaging [Chevrot and Girardin, 2000]. However, low-amplitude (e.g., comparable to that of mantle $P / S$ conversions) noise should be encountered far more frequently than the strong noise removed during data culling, and thus it is difficult to specify a welldefined threshold on the noise level retained in the data. Extensive record editing is prone to subjectivity and also defeats any possibilities for quality (coherency) control of the type described above.

[20] The above approach allows us to investigate the variability of the signal-to-nose ratio within the image, on a subset of the data used, as well on the frequency band. The method provides a direct assessment of the final RF image quality in 3-D, suitable for most imaging techniques employed to date. For large-aperture, migrating arrays such as proposed for the USArray program, this method could readily provide maps of image quality attributes, such as the $\mathrm{S} / \mathrm{N}$ ratios and CIG sampling. As our results suggest, this quality may vary significantly across short distances. With continuous monitoring of the RF image during array redeployment, such attributes could conceivably be used for real-time array tuning and depth image focusing.

[21] Acknowledgments. We are grateful to the IRIS PASSCAL program that made the data acquisition of the CD-ROM data set possible. Constructive comments by Sebastien Chevrot, Lupei Zhu, Stephane Rondenay, and the Associate Editor Jeroen Ritsema have greatly helped in improving the manuscript. This research was supported by NSF grant EAR99-03235. I.M. was also supported in part by the Defense Threat Reduction Agency grant DTRA01-01-C-0057.

\section{References}

Abers, G. A., Array measurements of phase used in receiver function calculations: Importance of scattering, Bull. Seismol. Soc. Am., 88, 313-318, 1998.

Al-Yahya, K., Velocity analysis by iterative profile migration, Geophysics, 54, 718-729, 1989.

Ammon, C. J., G. E. Randall, and G. Zandt, On the nonuniqueness of receiver function inversions, J. Geophys. Res., 95, 15,303-15,318, 1990.

Bostock, M. G., Ps conversions from the upper mantle transition zone beneath the Canadian landmass, J. Geophys. Res., 101, 8393-8402, 1996.

Bostock, M. G., and S. Rondenay, Migration of scattered teleseismic body waves, Ps, Geophys. J. Int., 137, 732-746, 1999.

Bostock, M. G., S. Rondenay, and L. Shragge, Multi-parameter 2-D inversion of scattered teleseismic body waves, 1 , Theory for oblique incidence, J. Geophys. Res., 106, 30,771-30,782, 2001.
Chevrot, S., and N. Girardin, On the identification of reflected and converted waves from receiver functions, Geophys. J. Int., 141, 801-808, 2000.

Chevrot, S., L. Vinnik, and J. P. Mintagner, Global-scale analysis of the mantle Ps phases, J. Geophys. Res., 104, 20,203-20,219, 1999.

Dueker, K. G., and A. F. Sheehan, Mantle discontinuity structure beneath the Colorado Rocky Mountains and High Plains, J. Geophys. Res., 103, 7153-7169, 1998.

Dueker, K., H. Yuan, and B. Zurek, Thick structured Proterozoic lithosphere of the Rocky Mountain region, GSA Today, 4(12), 3-9, 2001.

Gurrola, H., and J. B. Minster, Evidence for local variations in the depth to the $410-\mathrm{km}$ discontinuity beneath Albuquerque, New Mexico, J. Geophys. Res., 105, 10,847-10,856, 2000.

Gurrola, H., J. B. Minster, and T. Owens, The use of velocity spectrum for stacking receiver functions and imaging upper mantle discontinuities, Geophys. J. Int., 117, 427-440, 1994.

Langston, C. A., and K. Hammer, The vertical component $P$-wave receiver function, Bull. Seismol. Soc. Am., 91, 1805-1819, 2001.

Lewis, J. L., S. M. Day, H. Magistrale, R. Castro, L. Astiz, C. Rebollar, J. Eakins, F. L. Vernon, and J. N. Brune, Crustal thickness of the Peninsular Ranges and Gulf Extensional Province in the Californias, J. Geophys. Res., 106, 13,599-13,611, 2001.

Morelli, A., and A. M. Dziewonski, Topography of the core-mantle boundary and lateral homogeneity of the liquid core, Nature, 325, 678-683, 1987.

Neal, S. L., and G. L. Pavlis, Imaging $P$-to- $S$ conversions with multichannel receiver functions, Geophys. Res. Lett., 26, 2581-2584, 1999.

Poppeliers, C., and G. L. Pavlis, Three-dimensional, prestack, plane wave migration of teleseismic $P$-to- $S$ converted phases, 1, Theory, J. Geophys. Res., 108, doi:10.1029/2001JB000216, in press, 2003a.

Poppeliers, C., and G. L. Pavlis, Three-dimensional, prestack, plane wave migration of teleseismic $P$-to- $S$ converted phases, 2, Stacking multiple events, J. Geophys. Res., 108, doi:10.1029/2001JB001583, in press, 2003b.

Rietsch, E., Estimation of the signal-to-noise ratio of seismic data with an application to stacking, Geophys. Prospect., 28, 531-550, 1980.

Rondanay, S., M. G. Bostock, and J. Shragge, Multiplarameter two-dimensional inversion of scattered teleseismic body waves, 3, Application to the Cascadiadata 1993 set, J. Geophys. Res., 106, 30,795-30,807, 2001.

Shearer, P., Constraints on upper mantle discontinuities from observation of long-period reflected and converted phases, J. Geophys. Res., 96, $18,147-18,152,1991$

Sheehan, A. F., P. Shearer, H. J. Gilbert, and K. G. Dueker, Seismic migration processing of $P-S V$ converted phases for mantle discontinuity structure beneath the Snake River Plain, western United States, J. Geophys. Res., 105, 19,055-19,065, 2000.

Shen, Y., S. C. Solomon, I. T. Byarnason, and C. J. Wolfe, Seismic evidence for a lower-mantle origin of the Iceland Plume, Nature, 395, 62-65, 1998.

Vinnik, L. P., Detection of waves converted from $P$ to $S V$ in the mantle, Phys. Earth Planet. Inter., 15, 39-45, 1977.

Zhu, L., Crustal structure across the San Andreas Fault, southern California from teleseismic converted waves, Earth Planet. Sci. Lett., 179, $183-$ $190,2000$.

K. G. Dueker, Department of Geology and Geophysics, University of Wyoming, Laramie, WY 82071-3006, USA.

I. B. Morozov, Department of Geological Sciences, University of Saskatchewan, 114 Science Place, Saskatoon, Saskatchewan, Canada S7N 5E2. (igor.morozov@usask.ca) 